\title{
Effect of Single and Multiple ROI Coding on JPEG2000 Performance
}

\author{
Omprakash S. Rajankar \\ Ph.D. Research Scholar, MPSTME, NMIMS University, Mumbai, India \\ Email: online.omrajankar@gmail.com \\ Uttam D.Kolekar \\ Principal, A. P. Shah Institute of Technology, Thane, India \\ Email: uttamkolekar@gmail.com
}

\begin{abstract}
Images are an integral part of advertisements. Images make the web pages heavy. It increases the response time if the size of the image is large and or available bandwidth is low. The consequence of it is viewer may lose his interest in the particular advertisement if he has to wait for a longer time. Image compression is one of the solutions to this problem. In advertisement images, ROI is of prime importance. Though the context of ROI and background regions are not of prime importance, they cannot be totally discarded. This paper investigates the effect of ROI coding on JPEG2000 performance. It proposes Multiple ROI (MROI) coding for compression of natural and advertisement images at moderate compression ratio. The proposed MROI coding prioritizes ROI codeblocks according to the ROI importance, and contribution of ROI in the specific ROI codeblock. It improves fine-grain accuracy at codeblock level also efficiently utilize the given bit budget with a negligible increase in encoding time.
\end{abstract}

Index Terms-EBCOT, Implicit, JPEG2000, Maxshift, Multiple ROI, Post Compression Rate Distortion.

\section{INTRODUCTION}

The primary objective of the compression algorithm is to represent an input with a smaller number of bits. It saves both storage space and transmission time. It is a general observation that high degree image compression introduces noticeable degradation in the visual quality of decompressed image. The quality of image and compression are mutually opposite in nature, and one has to sacrifice for another. Further, in a picture, all regions are not equally important. Therefore, to provide higher reconstruction quality for the important region, Region of Interest (ROI), based compression methods are developed.

Human being tracks an image unevenly in free viewing or sequential manner. During this process, some of the locations are repeatedly visited and may hold viewer' attention for a longer duration because some of the locations are more attention grabbing. It leads to multiple ROI concept in image processing. If an image is compressed considering the multiple ROIs, it may be beneficial in two ways; one is the effective use of available bit budget and the second is eye pleasing image quality.

ROI coding is one of the essential features of JPEG2000 image compression standard. JPEG2000 part1 provides two mechanisms of ROI coding, referred as Maxshift method that modifies the scale of wavelet coefficients, and another is an Implicit method, that changes the rate-distortion factor to prioritize the ROI. Both the mechanisms have some pros and cons. Modifying wavelet coefficient mechanism achieves an excellent fine grain accuracy to delimit ROI areas. However, it fails to support multiple ROIs [1]. In the case of Maxshift at the low bit rate background (BG) is blurred, patchy and even absent sometimes, if the bit budget is exhausted in the reconstruction of ROI. So the overall effect is not eye pleasing. The Implicit method is Post Compression Rate-Distortion (PCRD) optimization based. It supports multiple ROIs with progressive, lossless mode. However, as it works at a codeblock level, it fails to delimit ROI areas finely. Further, the improvement in the ROI quality is too small and is hardly noticeable.

ROI coding depends on both JPEG2000 attributes and ROI parameters. The JPEG2000 attributes that affect ROI coding are wavelet filter types, decomposition levels, the number of quality layers and codeblock sizes. Similarly, ROI parameters like ROI sizes, ROI shapes, the number of ROIs, the weight of ROI codeblocks and the inclusion of low-resolution sub-band as an ROI affects the ROI coding performance. This paper discusses the effect of ROI coding on JPEG2000 performance. It also proposes and implements multiple ROI coding method in JPEG2000 framework for compression of natural images. Especially for advertisement image compression at moderate bitrate, the proposed methods are superior to existing Maxshift and Implicit ROI coding methods.

The plan of the paper is as follows. Existing JPEG2000 compliant ROI coding methods and proposed methods are discussed in Section II; Section III signifies the performance measures used for evaluation; Section IV Investigate Effect of ROI parameters on the performance of JPEG2000, and provides numerical and visual results assessing the performance of proposed multiple ROI coding methods. Section V concludes with remarks. 


\section{JPEG2000 COMPLIANT ROI CODING METHODS}

The ROI based compression can compress the image with higher resolution in ROI than the background. This section reviews the ROI coding methods for JPEG2000. The JPEG2000 standard defines only the decoder. Any encoder or compressor is JPEG2000 complaint if the generated code stream is decodable by JPEG2000.

\section{A. MAXSHIFT ROI coding method}

JPEG2000 part-1 supports Maxshift ROI method [2]. It can prioritize arbitrary ROI. It completely encodes/decodes the ROI before the background. Further, the ROI shape need not be transmitted to the decoder. In the case of color images, the method applies separately to each color component. The steps involved are as follows:

- Generation of mask: Let $M_{i}(m, n)$ be a binary mask for ROI, $i$, where

$$
M_{i}(m, n)=\left\{\begin{array}{llc}
1 & \text { inside } & \text { ROI } \\
0 & \text { outside } & \text { ROI }
\end{array}\right.
$$

- Determination of scaling value: The scaling value $s$ is chosen as the largest number of magnitude bitplanes, for any background coefficient in any codeblock.

- Scaling the coefficients: The Maxshift method scales up the coefficients associated with an ROI well above the background coefficients and eliminates the need for the mask at the decoder.

- Finally, the scaling value is written into the codestream along with the RGN marker, specified in JPEG2000 standard.

At receiver, the decoder can identify ROI coefficients from the scaling value in RGN marker and shifts them to their original value. It decodes ROI fully before it starts decoding the background data. It is the major limitation of Maxshift.

Bit-plane by Bit-plane Shift (BbBShift)[3] and Generalized Bit-plane by Bit-plane Shift ROI methods (GBbBShift) [4] aims to overcome this limitation; these methods are variants of Maxshift. Small modification in the decoder can make them compliant with JPEG 2000. The wavelet coefficients double in dynamic range to accommodate the arbitrary ROI shape in non-overlapping bit plane methods.

\section{B. Implicit ROI coding method}

The Post Compression Rate-Distortion Optimization algorithm (PCRD) [5] organizes each codeblock bit stream to minimize the distortion optimally while achieving the required bit rate constraints. ROI coding methods based on modifying the distortion estimation take advantage of the rate-distortion optimization stage. PCRD independently truncate the bit stream to a collection of segments $R_{i}^{n}$, that result in distortion of $D_{i}^{n}$ in the reconstructed image in such a way that most of these truncation points lie on the convex hull of the corresponding rate-distortion curve. For each truncation point, $n$, the relevant distortion, and rate are additive, i.e.

$$
D=\sum D_{i}^{n_{i}} \quad R=\sum R_{i}^{n_{i}}
$$

Where, $D$ denotes overall image distortion, and $R$ represents a rate. Equation (3) gives an additive distortion metric which approximates Mean Squared Error (MSE).

$$
\hat{D}_{i}^{n}=w_{b_{i}}^{2} \sum\left(\hat{s}_{i}[k]-s_{i}[k]\right)^{2}
$$

Here, $S_{i}[k]$ denotes subband samples in the codeblock $B_{i}, \hat{S}_{i}[k]$ denotes the quantized representation of these samples, $w_{b_{i}}$ is the L2 norm of the wavelet basis functions, to which codeblock belongs. To minimize distortion for a given bit rate, $R_{\max }$ and the optimal selection of the truncation points $n_{i}$,

$$
D(\lambda)+\lambda R(\lambda)=\sum\left(D_{i}^{n_{i}^{\lambda}}+\lambda R_{i}^{n_{i}^{\lambda}}\right)
$$

The Lagrange's multiplier $\lambda$ is optimized for which the distortion cannot be reduced further without increasing the overall rate and vice-versa.

ROI coding is possible with modification in PCRD. The codeblocks containing ROI coefficients are effectively more prioritized than codeblocks containing background coefficients. Major benefits of this method are: absolute priority to ROI over the background is not compulsory. Also, the ROI priority is not restricted to be a power of 2. Fig.1. shows the ROI codeblock prioritization methods based on modification in PCRD. A method based on this mechanism in JPEG 2000 is the Implicit ROI coding method [6]. The Implicit ROI coding method increases the coding pass distortion estimates for every codeblock whose coefficients contribute to ROI by a factor

$$
D_{i}^{y_{j_{k}}}=\left\{\begin{array}{c}
R_{w} * w_{b_{i}}^{2} \sum_{k \in B_{i}}\left(\hat{s}_{i}^{j_{k}}[k]-s_{i}[k]\right)^{2}, \text { ROIcodeblock } \\
w_{b_{i}}^{2} \sum_{k \in B_{i}}\left(\hat{s}_{i}^{j_{k}}[k]-s_{i}[k]\right)^{2}, \text { otherwise }
\end{array}\right.
$$

Fig. 1(b) illustrate the concept of Implicit ROI method, wherein it assigns the same weight to all the ROI codeblocks. The unwanted feature of the implicit method is that it only distinguishes ROI and the background codeblocks on a block by block basis. Also, the same priority is applied to all the ROI codeblocks, irrespective of a contribution of ROI coefficients in the codeblock. This grainy unfairness between codeblocks belonging to the ROI and background do not achieve fine-grain accuracy.

Other methods based on PCRD in literature are separated codeblock ROI coding method [7], ROI coding 
method based on EBCOT [8]. The separated ROI codeblock method splits the codeblocks into ROI and background. In ROI codeblock, all the background coefficients are zero and vice-versa. It encodes similar to MAXSHIFT method. In separated codeblock ROI coding method, it is not possible to combine ROI and background finely. ROI coding method based on EBCOT is similar to the Implicit method, where few least significant bit planes of the background wavelet coefficients are trimmed to change the priority of ROI codeblocks [5]. IMP-J2K [9] uses the same principle of reordering codeblock contributions to prioritize ROIs as an Implicit method and extend the framework described and implemented in [10] to accommodate the importance or saliency map to specify multiple ROIs and arbitrary importance scores.

\section{Weighted ROI Coding Method}

To improve the fine-grain accuracy weighted and subblock ROI coding methods are proposed in [11], that changes the distortion contributions of codeblocks according to the number of coefficients within the codeblock of an ROI. Equation (6) defines the distortion contributions.

$$
D_{i}^{\prime j_{k}}=\left\{\begin{array}{c}
R_{c} * w_{b_{i}}^{2} \sum_{k \in B_{i}}\left(\hat{s}_{i}^{j_{k}}[k]-s_{i}[k]\right)^{2}, \text { ROICB } \\
w_{b_{i}}^{2} \sum_{k \in B_{i}}\left(\hat{s}_{i}^{j_{k}}[k]-s_{i}[k]\right)^{2}, \text { otherwise }
\end{array}\right.
$$

Where

$$
R_{c}=C_{\text {count }} / \operatorname{sizeof}\left(B_{i}\right)
$$

Fig. 1(c) illustrate the concept of weighted ROI method, wherein ROI weights are proportional to the ROI codeblock contribution.

Both the methods discussed prioritize the codeblocks, but do not distinguish ROIs. The advantage of weight method is it tries to approximate the fine-grain accuracy of Maxshift method at codeblock level. To distinguish ROIs and utilize the given bit budget efficiently there is a need for multiple ROI coding.

\section{PROPOSED JPEG2000 COMPLIANT ROI CODING METHODS}

The ROI methods should prioritize more than one ROI at different priorities. They should take care of arbitrary shape multiple ROIs with or without overlapping. Further, they should provide fine grain accuracy like a coefficient scaling method without much affecting the coding efficiency. Following are the multiple ROI coding methods proposed, that modifies the Implicit methods, to partially fulfill the above requirements.

\section{A. Implicit multiple ROI coding method}

This method prioritizes the codeblocks as per the viewers interest in the particular region of the image. This method substitute ROI weight $R_{w}$ in equation (5) by the importance value $R_{v i}$, to prioritize the codeblock belonging to a specific ROI. Fig. 1(d) is a sketch to understand the concept of implicit multiple ROI coding method. It shows codeblocks belonging to different ROIs with different uniform weights within each ROI. The proposed method modifies the existing Implicit method to prioritize multiple ROIs.

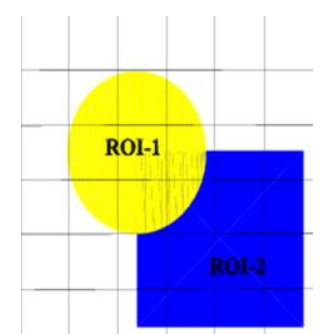

(a) Sample of image with multiple ROI

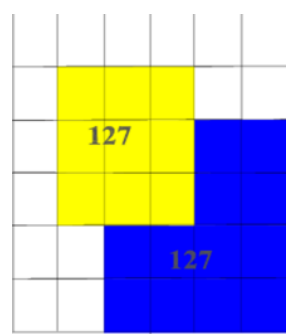

(b) Implicit method

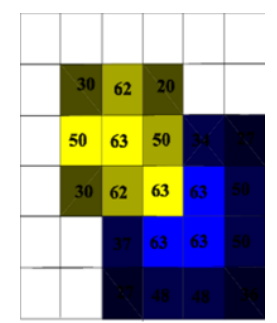

c) Weighted ROI codeblock Method

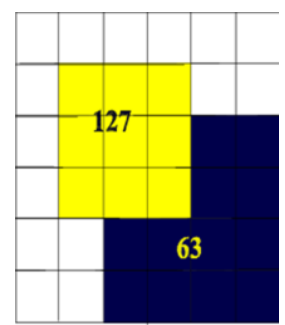

(d ) Implicit multiple ROI coding Method

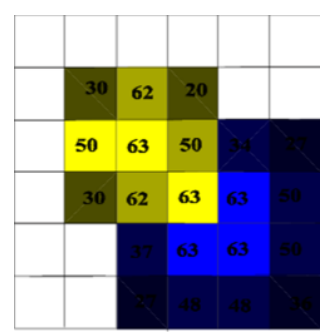

(e )Weighted multiple ROI coding Method

Fig.1. PCRD based ROI codeblock prioritization methods 


\section{B. Weighted multiple ROI coding method}

This method prioritizes the codeblocks not only as per the viewers interest in the particular region of the image but also as per ROI contribution in the codeblocks. This method substitute ROI weight $R_{w}$ by the product of importance value $R_{v i}$ and $\mathrm{ROI}$ contribution $R_{c i}$ to prioritize the codeblock belonging to a specific ROI as in (7)

$$
D_{i}^{\prime j_{k}}=\left\{\begin{array}{c}
R_{c_{i}} * R_{v_{i}} * w_{b_{i}}^{2} \sum_{k \in B_{i}}\left(\hat{s}_{i}^{j_{k}}[k]-s_{i}[k]\right)^{2}, \text { ROICB } \\
w_{b_{i}}^{2} \sum_{k \in B_{i}}\left(\hat{s}_{i}^{j_{k}}[k]-s_{i}[k]\right)^{2}, \text { otherwise }
\end{array}\right.
$$

Fig. 1(e) is a sketch to understand the concept of weighted multiple ROI coding method. The codeblocks in which more than one ROI appear gets a maximum importance value in that codeblock. Thus, the proposed

ROI method modifies the Weighted ROI method to prioritize multiple ROIs. It is an expectation that the finegrain accuracy of this method is better than the modified Implicit ROI method because it takes into consideration the ROI contribution in each codeblock in addition to the importance value.

\section{PERformance MeAsures}

The pixel difference, correlation, and edge detection decides the objective measures. In this work, both the original and reconstructed images are available therefore it is possible to define following full-reference objective quality measures.

\section{A. Peak Signal-to-Noise Ratio (PSNR)}

PSNR defined in (8) is the most widely used image distortion metric due to its computational simplicity. Large PSNR values indicate the small difference between the original and reconstructed image.

$$
P S N R=10 \log \frac{s^{2}}{M S E}
$$

Where, $\mathrm{s}=255$ for an image with bit-depth of 8 bits, and, the Mean Square Error (MSE):

$$
M S E=\frac{1}{N M} \sum_{i=0}^{N-1} \sum_{j=0}^{M-1}[f(i, j)-\hat{f}(i, j)]^{2}
$$

Where $f(i, j)$ and $\hat{f}(i, j)$ are the original and reconstructed images of size $N X M$. As the PSNR is slightly biased towards over smoothed or blurred images, one cannot rely solely on PSNR alone.

\section{B. Structural Similarity (SSIM) index}

In 2002, Wang and Bovik proposed Universal Image Quality Index (UIQI) measure [12], it breaks the comparison between original and distorted image into three comparisons: luminance, contrast, and structural comparisons. Wang and Bovik proposed SSIM as an improvement for UIQI. SSIM measures the similarity between two images. It is designed to be consistent with the human eye perception. The original and reconstructed images are divided into blocks (for example $8 \times 8$ ), and then, comparison measures luminance, contrast, and structure are computed from (10)-(12) respectively.

$$
\begin{aligned}
& l(f, \hat{f})=\frac{2 \mu_{f} \mu_{\hat{f}}+C_{1}}{\mu_{f}^{2}+\mu_{\hat{f}}^{2}+C_{1}} \\
& c(f, \hat{f})=\frac{2 \sigma_{f} \sigma_{\hat{f}}+C_{2}}{\sigma_{f}^{2}+\sigma_{\hat{f}}^{2}+C_{2}} \\
& s(f, \hat{f})=\frac{\sigma_{f \hat{f}}+C_{3}}{\sigma_{f} \sigma_{\hat{f}}+C_{3}}
\end{aligned}
$$

Where, mean, standard deviation and covariance are calculated from the original and reconstructed images. Finally, the SSIM is the product of luminance, contrast, and comparison structure measures. For $C_{3}=C_{2} / 2$ the SSIM is given by

$$
\operatorname{SSIM}(f, \hat{f})=\frac{\left(2 \mu_{f} \mu_{\hat{f}}+C_{1}\right)\left(2 \sigma_{\hat{f}}+C_{2}\right)}{\left(\mu_{f}^{2} \mu_{\hat{f}}^{2}+C_{1}\right)\left(\sigma_{f}^{2}+\sigma_{\hat{f}}^{2}+C_{2}\right)}
$$

Where, $C_{1-3}$ are the constants. SSIM takes into account the similarity of edges (high-frequency content) between the reconstructed image and the original one. Therefore, SSIM is a "better quality measure", but it is more complicated to compute than PSNR.

Subjective IQMs are better than objective IQMs, but they are time consuming and expensive. The objective IQM field is still open and needs lots of work to correlate with subjective IQM.

\section{PERFormance EVAluation}

The part-A of this section, conduct few experiments to investigate the effect of ROI and JPEG2000 parameters on the performance of JPEG2000. Specifically, the methods investigated are Implicit and Maxshift ROI coding methods. The part-B of this section, evaluate the proposed ROI coding method.

A. Investigation of Effect of JPEG2000 attributes and ROI parameters

\section{Effect of JPEG2000 attributes}

The investigation of the effect of different wavelet filters, decomposition levels, quality layers, and codeblock sizes is as follow.

i. FILTER TYPES: JPEG 2000 uses CDF 9/7 and integerinteger CDF $5 / 3$ wavelet filters. Fig. 2. shows 
original and reconstructed images of Maxshift ROI method with the two wavelet filters.

The interpolation based fast saliency detection method proposed in [13] is useful for the ROI detection. Table 1 lists the MSE of a reconstructed image for these filters. The MSE for $9 / 7$ filter is more than $5 / 3$ filter as the support length of the wavelet filter $9 / 7$ is more than $5 / 3$. It causes more leakage of the ROI coefficients into the background adjacent to the ROI. This effect is prominent in the case of Maxshift method at low bit rate.

ii. Decomposition levels: In JPEG2000 by default five decomposition levels are used. As discussed above, the overlapping of wavelet basis function causes the leakage of ROI coefficients into the background, when wavelet decomposition levels are more effect of leakage is noticeable.

iii. The number of quality layers: The use of multiple quality layers is beneficial in the progressive transmission and reconstruction of images. Single layer ROI encoding does not provide any gain in encoding the image at full quality. The overhead using multiple layers is negligible; however, it increases at low bit-rates.

iv. Codeblock size: JPEG 2000 Part 1 uses codeblock sizes that are same for all subbands and resolution levels. Fine grain accuracy improves in the Implicit method, with the smaller size of codeblock. Table 2 shows performance for an image at $0.5 \mathrm{bpp}$. It indicates that for the smaller size of the codeblock, PSNR is more. However, it takes a longer time to encode the image. Therefore, $32 \times 32$ is an optimum choice of codeblock size for ROI coding, instead of default size $64 \times 64$.

Table 1. MSE of the reconstructed image with CDF 5/3 and 9/7 filters for Image 224 from IMGSAL dataset.

\begin{tabular}{|c|c|c|c|}
\hline \multirow{2}{*}{$\begin{array}{c}\text { Bit } \\
\text { Rate }\end{array}$} & \multirow{2}{*}{ Algorithm } & \multicolumn{2}{|c|}{ MSE of ROI } \\
\hline & & 5/3 Filter & 9/7 Filter \\
\hline \multirow{2}{*}{0.1} & Implicit & 29.1 & 30.0 \\
\hline & Maxshift & 17.7 & 24.6 \\
\hline \multirow{2}{*}{0.2} & Implicit & 12.4 & 13.1 \\
\hline & Maxshift & 4.5 & 6.6 \\
\hline \multirow{2}{*}{0.5} & Implicit & 1.5 & 2.1 \\
\hline & Maxshift & 0.51 & 0.52 \\
\hline \multirow{2}{*}{1} & Implicit & 0.28 & 0.41 \\
\hline & Maxshift & 0.13 & 0.13 \\
\hline
\end{tabular}

Table 2. The performance of an image at $0.5 \mathrm{bpp}$ for different codeblock sizes for Image 224 .

\begin{tabular}{|c|c|c|c|c|}
\hline \multirow{2}{*}{$\begin{array}{c}\text { Code } \\
\text { Block } \\
\text { Size }\end{array}$} & \multirow{2}{*}{$\begin{array}{l}\text { Encod } \\
\text { e Time } \\
\text { (Sec.) }\end{array}$} & \multirow{2}{*}{$\begin{array}{c}\text { Decode } \\
\text { Time } \\
\text { (Sec.) }\end{array}$} & \multicolumn{2}{|c|}{ PSNR } \\
\hline & & & BG & ROI \\
\hline $16 \times 16$ & 1.12 & 0.25 & 30.18 & 31.99 \\
\hline $32 \times 32$ & 0.63 & 0.25 & 30.24 & 31.79 \\
\hline $64 \times 64$ & 0.53 & 0.24 & 30.27 & 31.41 \\
\hline
\end{tabular}

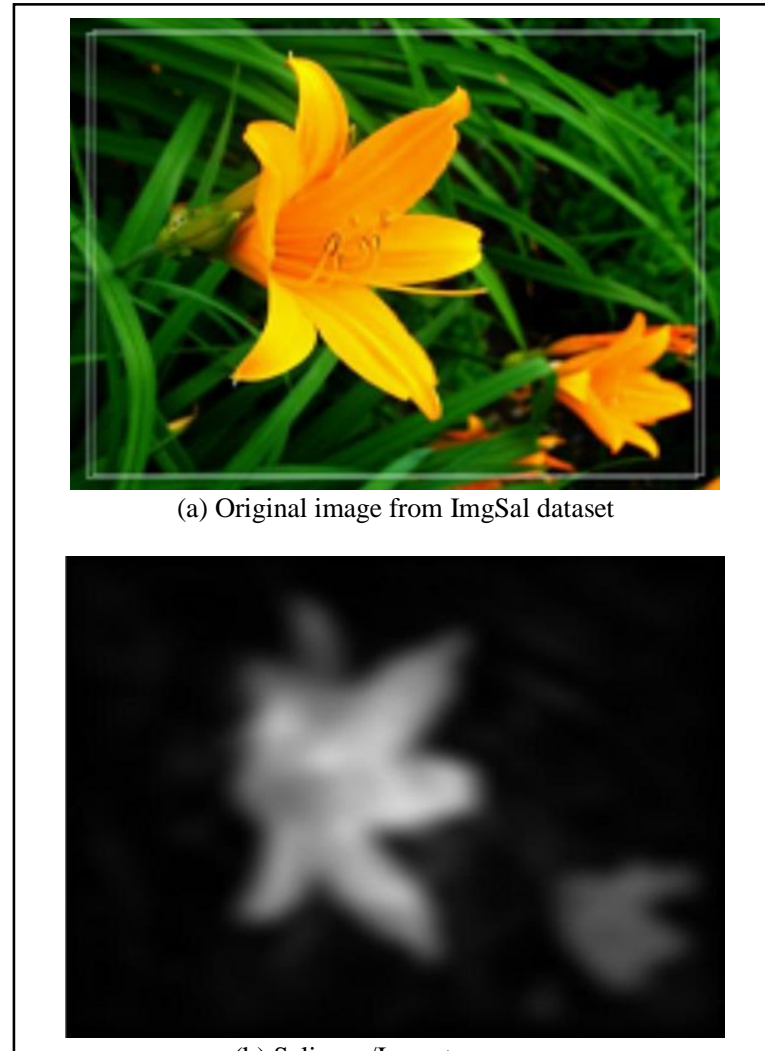

(b) Saliency/Importance map

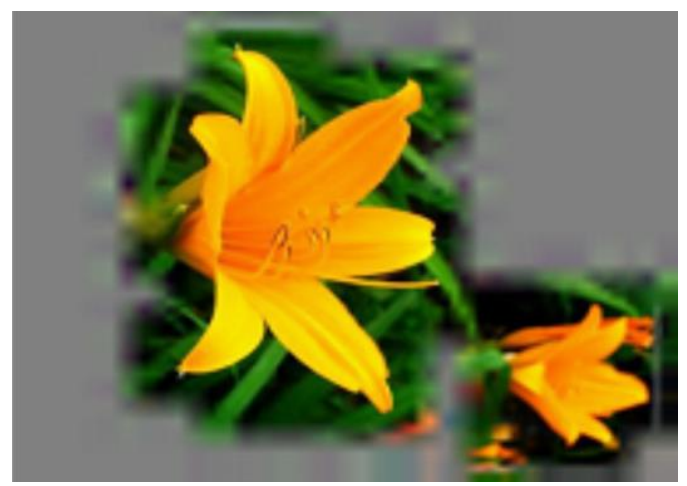

(c) Reconstructed with CDF 5/7 wavelet filter

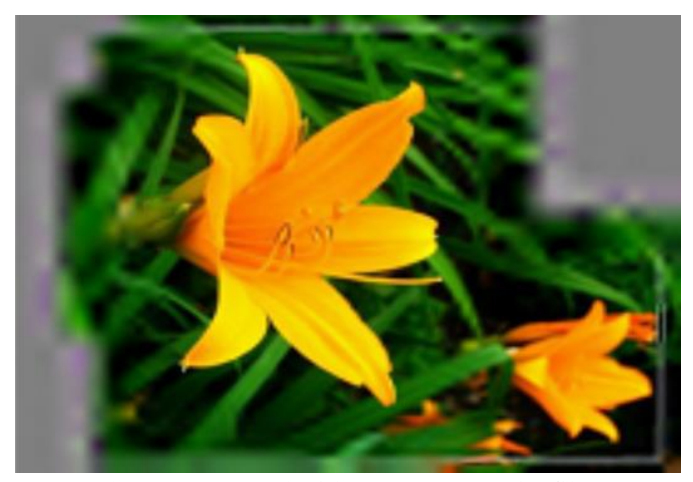

(d) Reconstructed with CDF 5/7 wavelet filter

Fig.2. Effect of filter length on the ROI compression with the Maxshift method.

1) Effect of ROI Parameters 
The effect of ROI parameters on the JPEG2000 image compression is investigated for ROI sizes, shapes, multiple ROIs and ROI weights.

i. ROI size: Max-shift requires increased numbers of bit-planes to distinguish ROI from the background. Further, increasing the ROI size increase the bits required to encode the ROI. ROI size up to $25 \%$ of the image area is recommended [14]. It is observed that the overheads for encoding ROIs in Max-shift supersede the benefits of ROI coding above this limit. If a large number of large ROIs were defined, then ROI performance degrades. However, the ROI size does not adversely affect the code-stream bit-rate for Implicit method

ii. ROI shape: Coefficient level ROI encoding is useful when encoding smaller and more complex shaped ROIs. Whereas block level ROI coding is helpful in large images and images in which the regions adjacent to the ROI are also of visual importance. All the ROI coding methods under consideration allow arbitrary ROI shapes. The effect of ROI shape is less important for the Max-shift since ROI encoding is at coefficient level.

iii. Number of ROIs: When there is a single cluster of attention points in an image, single ROI is sufficient, otherwise to efficiently compress the image, multiple ROIs with different priority are required to be defined. The standard implicit method can define Multiple ROIs. However, it assigns the same priority to all the ROIs. IMP-J2K, Weighted, and Sub-block methods are variants of the Implicit method. Thus, they provide the framework for multiple ROI prioritization. In the case of multiple ROIs, reconstruction of the image is progressive at full frame with the different ROIs reconstructed with higher quality than the rest of the image. Total ROI size limited to $25 \%$ and the number of ROIs up to 3 may result in improved ROI performance.

iv. The weight of ROI codeblocks $\left(R_{\text {weight }}\right)$ : Weight of ROI may be fix or proportional to the visual importance of the ROI. It is used to modify the distortion contributions in PCRD based Implicit ROI coding method. The background is of lowest priority, so weight assigned to the codeblocks belonging to the background is minimum; usually, its value is equal to one. In the case of the Implicit method the $R_{\text {weight }}$, more than 1 is assigned uniformly to all the codeblocks that contain at least one ROI coefficient. The distortion metric (MSE) of a codeblock belonging to ROI is modified by the square of the assigned $R_{\text {weight }}$ according to equation (5). Maxshift and scaling based methods use scaling of ROI coefficients by a scaling factor, $s$. There is a useful guideline that relates the $R_{\text {weight }}$ and $s$, provided the boundaries of ROI and codeblocks exactly coincide. Here, $s$ represents an equivalent number of bitplane shifts of ROI codeblocks. As an example, for Maxshift $s=14$ is equivalent to the square of $R_{\text {weight }}$ $\left(128^{2}\right)$ or $2^{14}$ for a hypothetical case in which the boundary of codeblocks and ROI coincide exactly. Fig. 3. shows the effect of $R_{\text {weight }}$ on the ROI and background coding in the Implicit method. When weight is one, the Implicit method performs just like JPEG2000 with no ROI emphasis. When $R_{\text {weight }}$ is 128 as per the guideline, PSNR-bit rate curve of Implicit should approach to that of the Maxshift method with maximum bitplane shift of 14 . This possibility is rare in the case of natural images, because of arbitrary shapes of ROIs. The general observation is, PSNR curves are cluttered in low as well as high-bit-rate, and ROI improve earlier than the background. It is an observation that the PSNR of ROI improves and PSNR of background decrease with an increase in $R_{\text {weight }}$ for Implicit as compared to JPEG2000 with no ROI. However, at low bitrate the improvement in PSNR of ROI is very much less as compared to Maxshift.

v. The inclusion of Low-Resolution Subband as an ROI: While improving the quality of ROI at low bitrate the background quality may degrade beyond identification in case of Maxshift ROI coding. Same thing happens at a low bitrate and high $R_{\text {weight }}$ for the Implicit method. In JPEG2000, ROI coding is facilitated with the ability to prioritize some degree of the background context by including the lowest subband of DWT decomposition in the ROI.

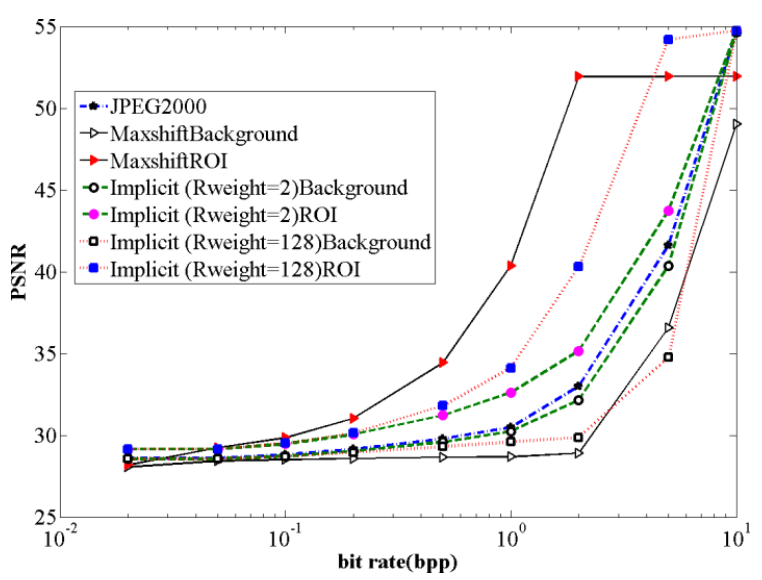

Fig.3. Comparison of JPEG2000, Maxshift and Implicit ROI coding at $R_{\text {weight }}=2$ and 128

\section{B. Investigation of JPEG2000 compliant Multiple ROI Coding Method}

Higher PSNR and SSIM represent better quality of the reconstructed image. The performance of the proposed ROI coding methods is assessed by comparing the PSNR and SSIM of these methods at different compression ratios with the standard JPEG2000, Maxshift and Implicit methods.

We implemented the methods in JPEG2000 Kakadu version 2.2.3 part-1 framework [15]. The overall size of ROI below $25 \%$ utilizes the bit budget efficiently. Also, the higher importance ROIs are of smaller sizes and viceversa. Fig. 4. compares the performance of the proposed methods with Maxshift, implicit and JPEG2000 at bit rate $0.05 \mathrm{bpp}$. Details of images are not visible in reduced size, 
so PSNR or PSNR difference with respect to JPEG2000 without ROI coding is useful. The degradation in PSNR greater than $2 \mathrm{~dB}$ is immediately noticeable. So, the patchy effect of Maxshift is visible in the Fig. 4(c). Depending on the availability of bit budget the ROIs and background are improved in progressive order from lossy to visually loss-less quality. Fig.5. and Fig.6. compare the PSNR and SSIM for different regions at different bit rates. At a moderate compression ratio (240:1 to $24: 1)$ or moderate bit rate ( 0.1 to $1.0 \mathrm{bpp}$ ), the proposed method shows overall better performance in ROI-1 to 3 and fair in the background region. At higher bit rates the PSNR and the SSIM differences gradually decreased. From the comparison of the performance of the proposed methods and the Maxshift method, it is apparent that the quality of the ROIs approaches to the quality of ROIs in Maxshift method while improving the background to such a level that the overall image quality is eye pleasing. Table 3 indicate that the time taken for encoding the image with the multiple ROI methods is less than the time taken by Maxshift method and negligibly greater than the Implicit method.
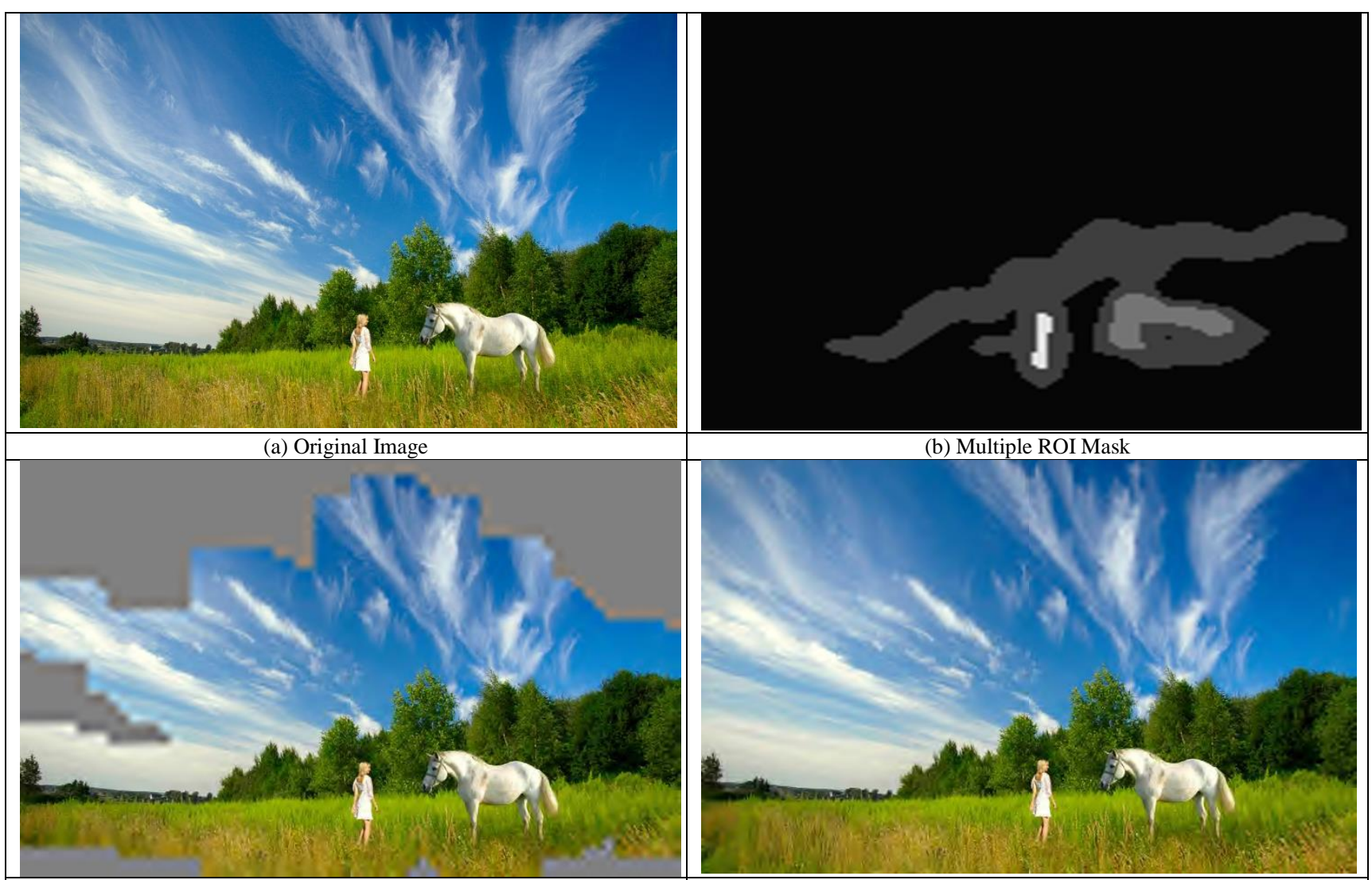

(b) Multiple ROI Mask
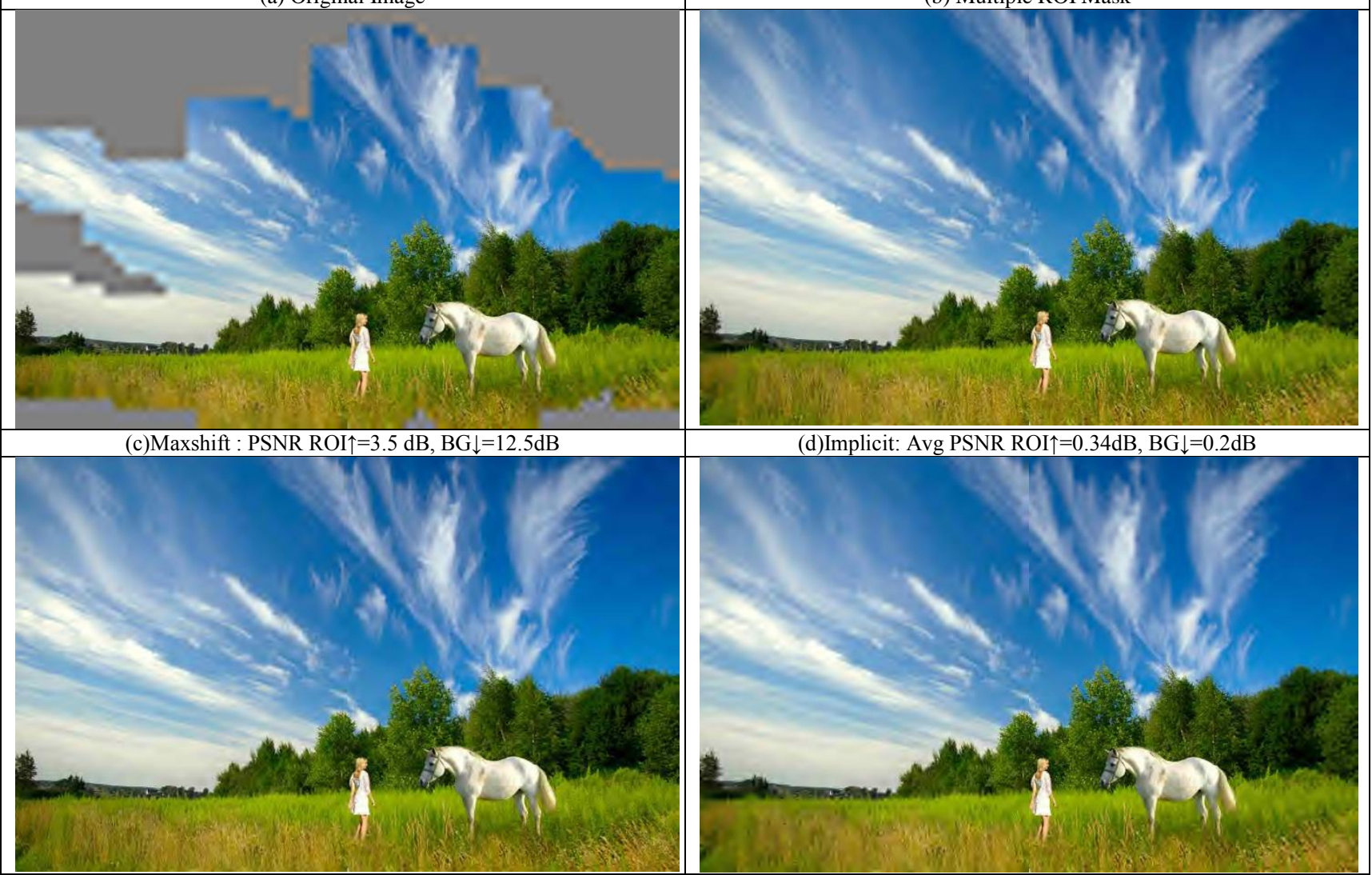

(d)Implicit: Avg PSNR ROI $\uparrow=0.34 \mathrm{~dB}, \mathrm{BG} \downarrow=0.2 \mathrm{~dB}$

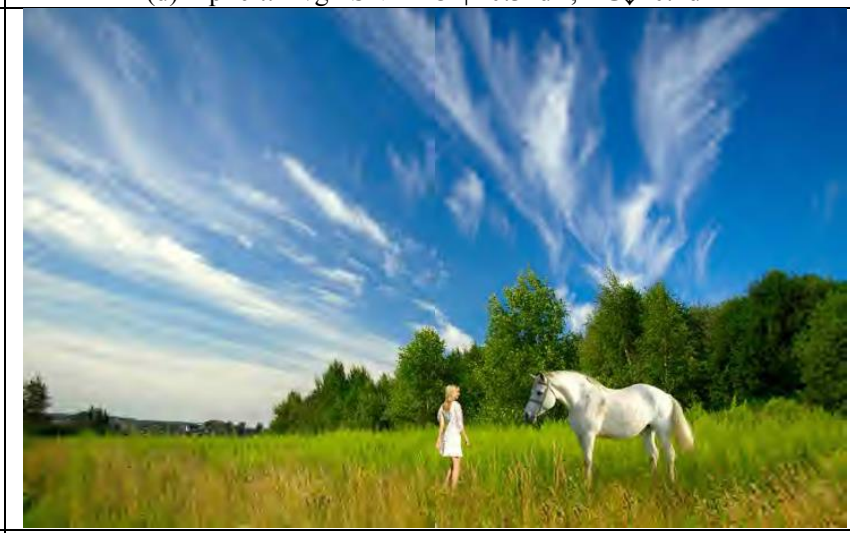

(e)Implicit Multiple: Avg PSNR ROI $\uparrow=2 \mathrm{~dB}, \mathrm{BG} \uparrow=1.2 \mathrm{~dB}$

(f) Weighted Multiple: Avg PSNR ROI $\uparrow=2.5$, BG $\downarrow=0.8 \mathrm{~dB}$

Fig.4. Results for the proposed method at 0.05 bpp bit-rates 


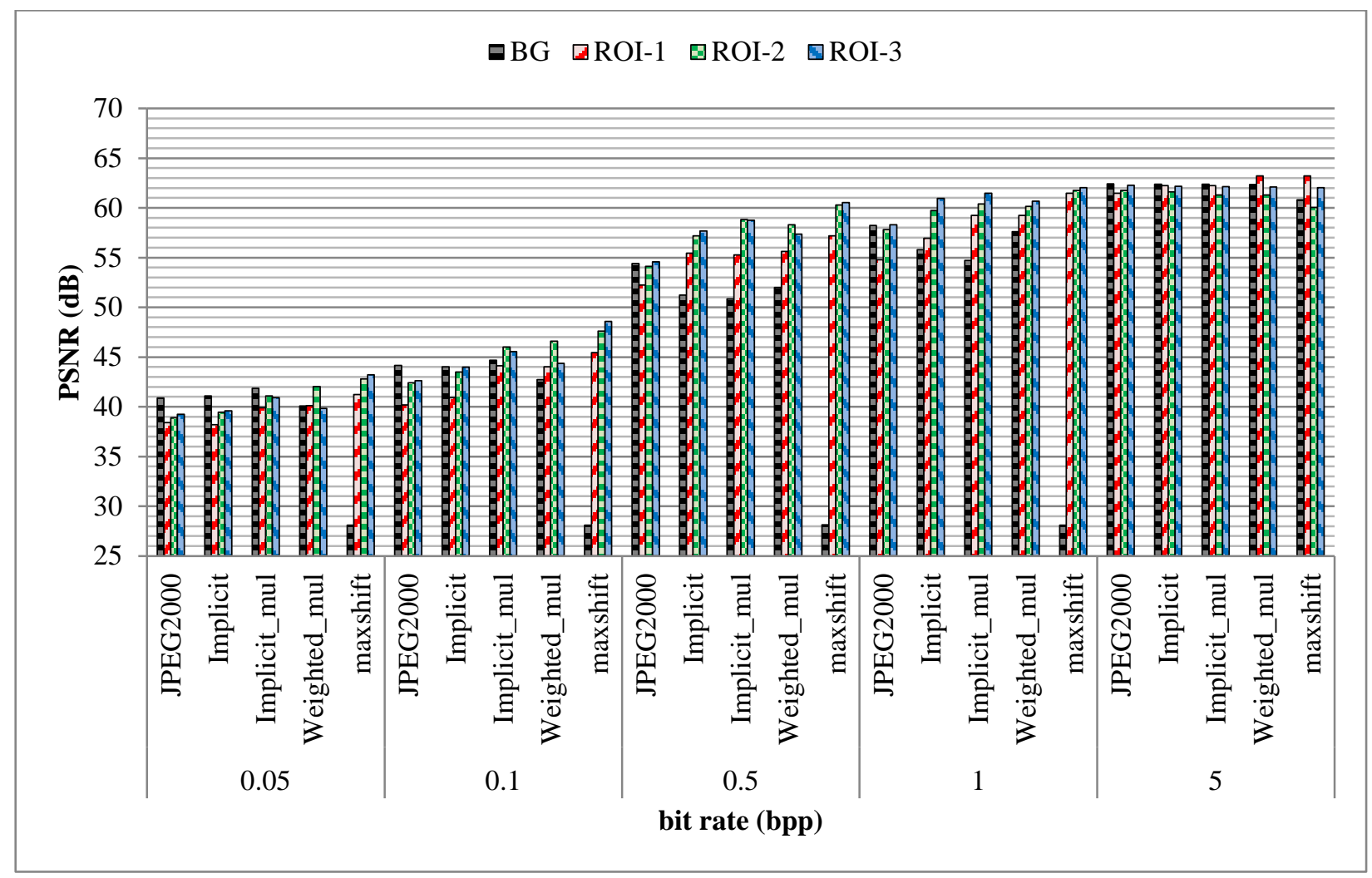

Fig.5. Results for the proposed method at different bit-rates: bit rate vs. PSNR

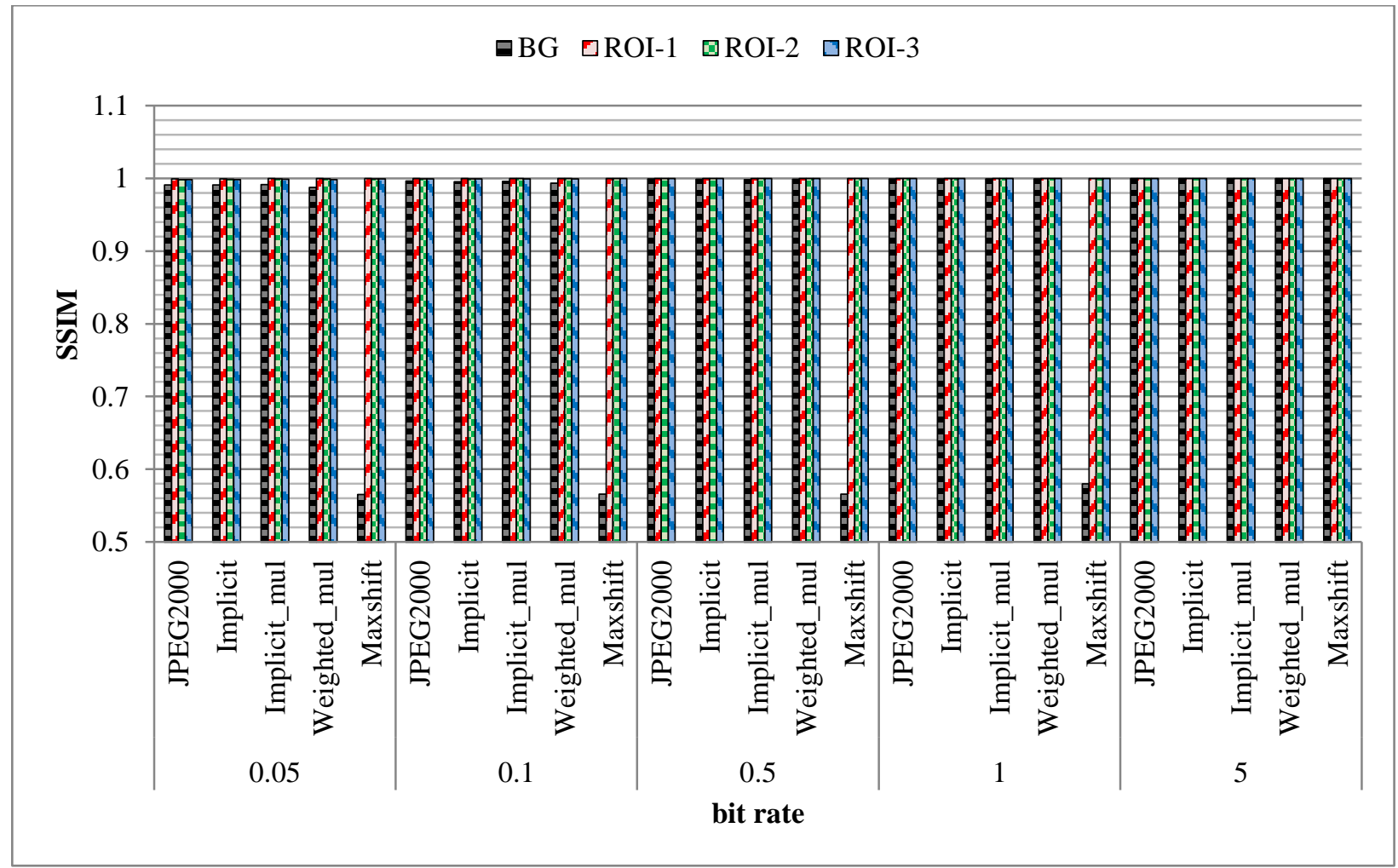

Fig.6. Results for the proposed method at different bit-rates: bit rate vs. SSIM 
Table 3. Encoding Time for different ROI methods

\begin{tabular}{|l|l|l|l|l|l|}
\hline $\begin{array}{c}\text { Bit } \\
\text { Rate }\end{array}$ & $\begin{array}{c}\text { Without } \\
\text { ROI }\end{array}$ & $\begin{array}{c}\text { Max- } \\
\text { shift }\end{array}$ & $\begin{array}{c}\text { Impli } \\
\text { cit }\end{array}$ & $\begin{array}{c}\text { Implicit- } \\
\text { Multiple }\end{array}$ & $\begin{array}{c}\text { Weighted- } \\
\text { Multiple }\end{array}$ \\
\hline 0.05 & 1.5 & 2.1 & 2.0 & 2.0 & 2.0 \\
\hline 0.1 & 1.5 & 2.1 & 1.9 & 2.0 & 2.0 \\
\hline 0.2 & 1.6 & 2.2 & 2.0 & 2.1 & 2.0 \\
\hline 0.3 & 1.6 & 2.2 & 2.1 & 2.1 & 2.1 \\
\hline 0.5 & 1.6 & 2.3 & 2.1 & 2.2 & 2.1 \\
\hline 1 & 1.7 & 2.7 & 2.3 & 2.3 & 2.3 \\
\hline 2 & 2.0 & 3.2 & 2.5 & 2.6 & 2.4 \\
\hline 5 & 2.3 & 3.7 & 2.9 & 2.9 & 2.9 \\
\hline
\end{tabular}

\section{CONCLUSION}

The fine grain accuracy for ROIs and encoding time in PCRD optimization based methods depends on the codeblock size. Small codeblock size provides fine grain accuracy; however it makes encoding slow. Therefore, an optimum choice of codeblock size for ROI coding of any image is $32 \times 32$ instead of $16 \times 16$ or default size $64 \times 64$.

In the case of coefficient level ROI methods, overheads of ROI coding increases with increase in ROI size. It degrades the ROI performance. However, the ROI size does not adversely affect the code-stream bit-rate of PCRD optimization technique. For small images with tiny arbitrary shape ROI, coefficient level ROI coding is a better choice. Whereas, PCRD optimization techniques provide better results for large size images with equally meaningful context and small sized codeblocks.

The support length of selected wavelet filter and decomposition levels affects the leakage of ROI in the adjacent background. Larger the support length of the wavelet filter and decomposition level, higher is the leakage of ROI in the adjacent background. Conversion of his limitation into an advantage by the proposed multiple ROI methods is possible. The proposed method distributes the given bit budget in a gradually decreasing order from ROI-1, ROI-2... down to the background.

The proposed multiple ROI coding method is JPEG2000 compliant. This method tries to preserve ROI and background. It progressively improves the quality of ROIs and background according to the priority at moderate bit rates. It approaches the fine-grain accuracy of the Maxshift method in the ROIs along with a smooth background as an implicit method at the same encoding time as an Implicit method, Thus, the proposed MROI efficiently compress an image and provide overall eye pleasing compressed image at moderate ( 0.1 to $1 \mathrm{bpp}$ ) bit rate.

\section{REFERENCES}

[1] O. Rajankar and U. Kolekar, "Generalized Multiple ROI Prioritization in JPEG2000 for Natural Images," vol. 6, no. 2, pp. 1636-1642, 2015.

[2] R. Grosbois, D. Santa-Cruz, and T. Ebrahimi, "New approach to JPEG 2000 compliant Region Of Interest coding," in SPIE's 46th annual meeting, Applications of Digital Image Processing XXIV, 2001, vol. 4472, pp. 267275.

[3] Z. Wang, S. Member, and A. C. Bovik, "Bitplane-byBitplane Shift ( BbBShift )— Image Coding," Signal Process. Lett. IEEE, vol. 9, no. 5, pp. 2000-2002, 2002.

[4] Z. W. Z. Wang, S. Banerjee, B. L. Evans, and a. C. Bovik, "Generalized bitplane-by-bitplane shift method for JPEG2000 ROI coding," Proceedings. Int. Conf. Image Process., vol. 3, pp. III-81,III-84 vol3,2002, 2002.

[5] D. Taubman, "High performance scalable image compression with EBCOT," IEEE Trans. Image Process., 2000.

[6] D. S. Taubman and M. W. Marcellin, JPEG 2000: Image Compression Fundamentals, Standards and Practice. Norwell, MA, USA: The Kluwer International Series in Engineering and Computer Science., 2001.

[7] Y. X. Y. Xie and G.-Q. H. G.-Q. Han, "ROI coding with separated code block," 2005 Int. Conf. Mach. Learn. Cybern., vol. 9, no. August, pp. 18-21, 2005.

[8] H. Yang, M. Long, and H.-M. Tai, "Region-of-interest image coding based on EBCOT," IEE Proceedings Vision, Image, and Signal Processing, vol. 152, no. 5. p. 590, 2005.

[9] A. N. Nguyen, "Importance Prioritised Image Coding in JPEG 2000," Queensland University of Technology, 2005.

[10] D. S. Taubman, M. W. Marcellin, and M. Rabbani, "JPEG2000: Image Compression Fundamentals, Standards and Practice," Journal of Electronic Imaging. 2002.

[11] J. Bartrina-Rapesta, J. Serra-Sagristà, F. Aulí-Llinàs, and J. M. Gómez, "JPEG2000 ROI coding method with perfect fine-grain accuracy and lossless recovery," in Conference Record - Asilomar Conference on Signals, Systems and Computers, 2009, vol. 16, no. 1, pp. 558-562.

[12] Z. Wang and A. C. Bovik, "A universal image quality index," IEEE Signal Process. Lett., vol. 9, no. 3, pp. 8184, 2002.

[13] O. S. Rajankar and U. D.Kolekar, "Scale Space Reduction with Interpolation to Speed up Visual Saliency Detection," Int. J. Image, Graph. Signal Process., vol. 7, no. 8, pp. 58-65, Jul. 2015.

[14] A. P. Bradley, F. W. M. Stentiford, I. Abradleyunsweduau, and F. W. M. S. Fredstentifordbtcom, "JPEG 2000 and Region of Interest Coding," no. January, pp. 303-308, 2002.

[15] M. Boliek, C. Christopoulos, and E. Majani, "JPEG 2000 Part I Final Committee Draft Version 1.0,” 2000.

\section{Authors' Profiles}

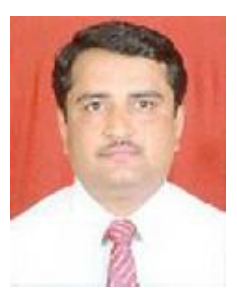

Dr. Uttam Kolekar received a Ph.D. degree from the Bharathi Vidyapeeth University, Pune in Electronics Engineering in 2008. He has been working as Principal at A. P. Shah Institute of Technology, Thane. He has total 23 years teaching experience at various posts such as Lecturer Assistant Professor, Professor, Head of Department, Dean and Director at various Institutes of Mumbai University. He is a research guide in different universities in India, and more than five candidates are working under him as research scholars from various Institutes and Industries like TCS. 
He has more than 15 papers in national and international Journals and conferences against his name. He is a reviewer for IEEE Transactions and Springer. He is a fellow member of (IETE), Life member of ISTE \& ISNT also he is a senior member of international association of Computer science \& information technology. He conducts seminars on mobile Adhoc network, Mobile computing, GSM, GPRS, and UMTS. He is an active reviewer for many international conferences. He has addressed many workshops on wireless networks, Sensors networks, and Cybersecurity.

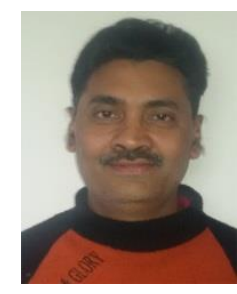

Mr.Omprakash Rjankar received M.E. (Electronics Engineering specialization in Computer ) degree from SRTM University, Nanded, India, in 2010. He is pursuing Ph.D from NMIMS University, Mumbai India. He has 26 years of experience in teaching at various posts such as Lecturer, Head of Department and Principal at different institutes under MSBTE in Mumbai and Pune. His areas of interest are Image Processing and VLSI. He has seven papers in International Conferences/journal to his credit. $\mathrm{He}$ is a life member of ISTE and student member of IEEE.

How to cite this paper: Omprakash S. Rajankar, Uttam D.Kolekar,"Effect of Single and Multiple ROI Coding on JPEG2000 Performance", International Journal of Image, Graphics and Signal Processing(IJIGSP), Vol.8, No.4, pp.2938, 2016.DOI: $10.5815 /$ ijigsp.2016.04.04 